\title{
Prevalencia y factores demográficos y laborales asociados al burnout de psiquiatras en Colombia*
}

\author{
Prevalence and both Demographic and Work Factors
} Associated with Burnout of Psychiatrists in Colombia

Recibido: junio 15 de 2010 | Revisado: enero 20 de 2011 | Aceptado: noviembre 1 de 2011

\author{
PaOla TejadA ** \\ VIVIOLA GÓMEZ ***
}

Universidad de Los Andes, Colombia

\section{RESU MEN}

La presencia de burnout puede afectar el bienestar de los médicos y la calidad del cuidado que brindan. El propósito de este estudio fue medir la prevalencia de burnout en los psiquiatras en Colombia, y determinar si su presencia está asociada con condiciones demográficas y otras variables laborales. Se realizó un estudio descriptivo transversal, encontrando que $9.9 \%$ de los participantes presentaban altos niveles de burnout. La edad, el trabajo como empleado y la dedicación exclusiva al área clínica fueron los principales factores asociados a la presencia del síndrome en esta población. Debe destacarse el uso por primera vez en Colombia del instrumento del CESQT, el cual mostró propiedades psicométricas adecuadas, lo que indica que puede usarse para evaluar el síndrome de burnout.

Palabras clave autores

Burnout, psiquiatras, prevalencia, variables demográficas, condiciones laborales. Palabras clave descriptores

Psicología de la salud, Cuestionario para la Evaluación del Síndrome de Quemarse por el Trabajo (CESQT), Colombia.

Para citar este artículo. Tejada, P. \& Gómez, V. (2012). Prevalencia y factores demográficos y laborales asociados al burnout de psiquiatras en Colombia. Universitas Psychologica, 11 (3), 863-873.

" Artículo de investigación.

*** Médico psiquiatra MSc. E-mail: paola_andreat@ hotmail.com

***** Profesora Asociada, Facultad de Ciencias Sociales, Departamento de Psicología. E-mail: vgomez@ uniandes.edu.co

\section{A B S T R A C T}

Burnout can affect not only wellbeing of medical professionals but also the quality of care they can provide to their patients. Aims of the study were to assess prevalence of burnout in Colombian psychiatrist and determine the associations of experiencing burnout with other demographic and working conditions. Transversal descriptive study showed that $9.9 \%$ of the participants were experiencing high levels of burnout. Age, being employed and working exclusively with patients were the principal predictors of burnout in this population. This study showed the good psychometric properties of the CEQST that invite to use it to assess the syndrome in this country. Key words authors

Burnout, psychiatrist, prevalence, demographic variables, working conditions. Key words plus

Health psychology, Evaluation Questionnaire Burnout (CESQT), Colombia. 


\section{Introducción}

El burnout es una consecuencia del estrés laboral crónico. En el caso del área médica, las manifestaciones de este síndrome pueden afectar no sólo el bienestar de los médicos sino también la calidad del cuidado brindado al paciente (Visser, Smets, Oort $\&$ de Haes, 2003).

En la actualidad existe una gran variedad de estudios sobre burnout, que si bien son útiles para la comprensión del problema, presentan varias limitaciones. Por una parte, abundan los estudios sobre burnout en profesionales del área de la salud, principalmente en enfermeras y en menor medida en médicos, pero este síndrome en psiquiatras ha sido poco estudiado, lo que ocurre también en Colombia, donde incluso se desconoce la prevalencia del problema en general y en profesiones específicas.

\section{Burnout}

Este concepto fue acuñado por primera vez por Freudenberger en 1974, pero su mayor desarrollo se logró con el trabajo de Maslach en 1982 (Salston \& Figley, 2003). Es un síndrome que se caracteriza por un estado progresivo de fatiga y/o apatía, e involucra una respuesta a estresores crónicos en el lugar de trabajo como resultado de una incongruencia entre capacidades, recursos o valores del trabajador y su empleo (Maslach, 2003).

El burnout es un síndrome compuesto por tres dimensiones: actitudes y sentimientos negativos hacia las personas con las que se trabaja (despersonalización), y hacia el propio rol profesional (falta de realización personal), así como por la vivencia de encontrarse emocionalmente agotado (desgaste emocional) (Illera, 2006).

Entre las causas del burnout se han mencionado en la literatura las siguientes (White, Edwards \& Townsend-White, 2006):

1. Factores individuales (expectativas sobrevaloradas con respecto al trabajo).

2. Factores interpersonales (diferencias entre los recursos del profesional y las demandas).
3. Naturaleza del trabajo (labores que implican cuidado de otros o con alta carga emocional).

4. Factores organizacionales (falta de apoyo, excesivas demandas cuantitativas).

El burnout también puede ser causado por conflictos entre valores individuales y demandas organizacionales, sobrecarga de responsabilidades, sensación de no tener el control sobre la calidad de los servicios prestados, una pobre compensación emocional o financiera, y la existencia de inequidad o falta de respeto en el lugar de trabajo (Salston \& Figley, 2003), fenómeno que ha sido descrito no sólo en los profesionales del área de la salud, sino también en una amplia gama de profesiones como personal de rescate, policías, enfermeras, profesores, etc. (Maslach, 2003; Trippany, White \& Wilcoxon, 2004).

Las explicaciones psicológicas asumen que en muchas de estas ocupaciones los roles implican consejos, protección y curación, asociadas a las demandas de aquellos que están bajo su cuidado. A pesar de los múltiples estudios que se han realizado sobre burnout hay puntos que no son claros, por ejemplo, definir si el burnout es únicamente una consecuencia del estrés laboral o si es el resultado de complejas interacciones entre factores sociales e individuales (Weber \& Jaekel-Reinhard, 2000).

De acuerdo con lo descrito en los párrafos anteriores, podemos acercarnos al concepto aduciendo que un desbalance entre los estresores psicosociales, laborales y la tolerancia individual al estrés son decisivos para el desarrollo del burnout. Siguiendo los planteamientos previamente descritos para la generación del estrés, esto parece indicar que el riesgo de presentar burnout está dado no sólo por la extensión de los factores estresantes y el déficit en los recursos personales, sino también por los sistemas de soporte social y las estrategias de afrontamiento disponibles. Adicionalmente a estilos de personalidad específicos tales como idealismo, perfeccionismo, timidez, inseguridad e inestabilidad emocional, otros factores negativos que influyen la tolerancia individual al estrés son estrategias pobres o inexistentes para manejarlo y un inadecuado soporte social (Weber \& Jaekel-Reinhard, 2000). 
Maslach (2003) afirma que, dado que el trabajo ocurre en un contexto interpersonal, deben estudiarse las transacciones que las personas realizan con los demás (pacientes y colegas), contexto interpersonal que enfoca la atención en las emociones del individuo y los motivos y valores subyacentes a su trabajo, y a los beneficiarios de su labor. Refiere que para su estudio hay seis áreas estratégicas de análisis (Maslach \& Leiter, 2003):

1. Carga de trabajo: la sobrecarga de trabajo tanto a nivel cualitativo como cuantitativo disminuye la capacidad de las personas para enfrentarse a las demandas laborales. La sobrecarga se ha relacionado consistentemente con el burnout, principalmente con la dimensión de desgaste emocional.

2. Control: Cuando las personas tienen más control en su trabajo pueden sentirse más satisfechos y comprometidos con su labor. La falta de control que lleva a conflicto en los roles del trabajador se asocia a burnout.

3. Recompensas: las recompensas intrínsecas (ej.: sentirse orgulloso por haber hecho algo bien o importante) son tan importantes como las recompensas extrínsecas (monetarias o sociales), pues al ser insuficientes aumentan la vulnerabilidad al burnout.

4. Rompimiento de redes de vínculo con la comunidad: tanto la comunidad laboral como familiar brindan asistencia emocional e instrumental, y reafirman la pertenencia de las personas a grupos significativos. Algunos empleos producen aislamiento de las personas con respecto a estos grupos, llevando a consecuencias negativas para los trabajadores.

5. Ausencia de justicia: se refiere a la percepción de que las decisiones en el trabajo son justas y el trabajador es tratado con respeto. Los empleados que sienten que sus jefes son justos son menos susceptibles a desarrollar burnout y a aceptar los cambios organizacionales.

6. Conflicto de valores: los valores hacen referencia a los ideales y valores que han acercado a las personas a sus trabajos. Entre mayor sea la brecha entre los valores individuales y los organizacionales se produce malestar y menor compromiso con el trabajo, hecho que ha sido asociado con las 3 dimensiones del burnout.

\section{Medición del burnout}

Dentro de los instrumentos psicométricos más utilizados en la actualidad y el que más se reporta en los estudios se encuentra el Maslach Burnout Inventory (MBI). Se trata de un cuestionario constituido por 22 preguntas en una escala tipo Likert que evalúa los sentimientos y actitudes del profesional en su trabajo, y en particular hacia los pacientes, midiendo los tres componentes del síndrome: desgaste emocional, despersonalización y falta de realización personal. No obstante, se ha descrito que este instrumento presenta insuficiencias psicométricas que son más notables cuando se adapta a diferentes idiomas, de manera que en algunos estudios es cuestionable la fiabilidad y validez de sus escalas tanto en adaptaciones al español como a otros idiomas (Gil-Monte, García-Juesas, Núñez, Carretero, Roldán \& Caro, 2006; Halbesleben \& Demerouti, 2005). Dentro de estas dificultades se incluye que la escala de despersonalización presenta con frecuencia valores Alfa de Cronbach bajos, en especial cuando el MBI se aplica fuera de EE.UU. y a muestras que no son de habla inglesa, lo que cuestiona la fiabilidad de los resultados obtenidos para esta variable, y por extensión los resultados obtenidos con el MBI (Gil-Monte, Carretero, Roldán \& Núñez-Román, 2005).

Otra de las dificultades encontradas se debe a que cuando se utiliza el MBI no existe un criterio unánime entre los expertos sobre el procedimiento para realizar el diagnóstico ni sobre los porcentajes de prevalencia e incidencia del síndrome (Gil-Monte et al., 2005), lo cual hace que sea particularmente difícil su uso en trabajos que intenten establecer grupos de riesgo (Gil-Monte et al., 2006).

En Colombia el MBI fue validado en el 2004 en la Universidad de Antioquia, donde se obtuvo una consistencia interna de 0.62 para agotamiento emocional, 0.72 para despersonalización y 0.76 para realización personal (Restrepo, Colorado \& 
Cabrera, 2006), valores que claramente muestran niveles bajos de confiabilidad, especialmente el correspondiente a agotamiento emocional.

Dadas las dificultades mencionadas en la literatura, se han creado instrumentos alternativos con el fin superar estas limitaciones, como el Copenhagen Burnout Inventory, CBI (Kristensen, Borritz, Villadsen \& Christensen, 2005), el Oldenburg Burnout Inventory, OBI (Halbesleben \& Demerouti, 2005) y el Cuestionario para la Evaluación del Síndrome de Quemarse por el Trabajo, CESQT (Gil-Monte et al., 2005). Este último será el instrumento a utilizar en la presente investigación, ya que se han obtenido resultados sólidos sobre su validez y fiabilidad con profesionales de la salud en muestras de España, Argentina, México y Chile (Gil-Monte \& Olivares, 2007; Marucco, Gil-Monte \& Flamenco, 2008; Unda, Sandoval \& Gil-Monte, 2008).

\section{Incidencia y prevalencia del burnout}

Respecto a la incidencia y prevalencia del burnout en población trabajadora, en los diferentes estudios se encuentra un porcentaje estadísticamente significativo de profesionales afectados. Illera (2006) revisó estudios y resultados epidemiológicos del síndrome de burnout, encontrando prevalencias que oscilan entre un $12 \%$ y un $50 \%$ en diferentes profesiones, mientras que en los estudios llevados a cabo con médicos se reporta entre un 30\% a $50 \%$. En uno de los pocos estudios realizados en Colombia, exclusivamente con población médica (Guevara, Henao \& Herrera, 2004), el burnout de intensidad entre moderada y severa se observó en el 85.3\% de los médicos incluidos en el estudio, lo cual llama la atención porque es una prevalencia muy alta comparada con la de los estudios mencionados. En el estudio realizado por Korkeila, Töyry, Kumpulainen, Toivola y Räsänen (2003), en la comunidad médica de Finlandia, se encontró que los psiquiatras tendían a reportar burnout moderado a severo más comúnmente que los otros médicos (55\% vs. 47\%), siendo importante aclarar que todos estos estudios han sido realizados con el Maslach Burnout Inventory (MBI). Para algunos autores estos datos de prevalencia son demasiado altos y se deben a una utilización incorrecta del instrumento (Marucco et al., 2008). Como se mencionó anteriormente, al no existir un criterio unánime sobre los puntos de corte que diferencian a quienes tienen burnout de aquellos que no lo tienen cuando se utiliza el MBI, los estudios que lo usan para expresar porcentajes de prevalencia presentan datos que no son comparables entre sí porque utilizan diferentes formas para realizar el diagnóstico (percentiles en algunos casos, puntos de corte en otros).

Estudios de prevalencia realizados con el Cuestionario para la Evaluación del Síndrome de Quemarse por el Trabajo (CESQT) han mostrado los siguientes datos: $11.7 \%$ en educadores de personas discapacitadas (Gil-Monte et al., 2005), 7.76\% en profesionales de atención a clientes (Gil-Monte \& Olivares, 2007), 5.09\% en trabajadores en cajas de compensación (Olivares \& Gil-Monte, 2007), y $3.25 \%$ en pediatras (Marucco et al., 2008).

Hoy en día se considera que el burnout debe ser tratado como un problema de salud pública (CebriàAndreu, 2005; Noblet, 2003) debido a su prevalencia y a las consecuencias negativas para quien lo padece y para las personas que están atendidas por quien presenta este síndrome. Cebrià-Andreu (2005) señala que:

Se puede afirmar que el síndrome de desgaste profesional se está convirtiendo en un problema de salud pública. Si los cuidadores se sienten mal cuidados o están exhaustos, es lógico pensar que, al final, quien pierde es la sociedad en su conjunto. En consecuencia, se debería considerar el bienestar de los médicos, y en general todas las profesiones de ayuda, una prioridad social. Simplemente por una cuestión de inteligencia (p. 470).

Dicho todo lo anterior, los objetivos de este estudió fueron medir la prevalencia de burnout en los psiquiatras en Colombia, y determinar si la presencia del síndrome en ellos está asociada con condiciones demográficas y laborales.

Los resultados de este estudio respecto a la prevalencia y a los factores de riesgo y protectores en profesionales de la salud mental producirán un conocimiento que puede ser útil para los terapeutas mismos, sus pacientes, y para las personas responsa- 
TABLA 1

Características demográficas y laborales de los participantes

\begin{tabular}{llcc}
\hline & & Frecuencia & Porcentaje \% \\
\hline \multirow{2}{*}{ Sexo } & Hombres & 68 & 56.2 \\
& Mujeres & 53 & 43.8 \\
\hline \multirow{3}{*}{ Estado civil } & Casado & 67 & 55.4 \\
& Soltero & 33 & 27.3 \\
& Unión libre & 13 & 10.7 \\
& Separado & 7 & 5.8 \\
& Viudo & 1 & 0.8 \\
\hline \multirow{2}{*}{ Tiempo de ejercicio de la profesión } & 1 a 5 años & 33.1 \\
& 6 a 10 años & 40 & 24 \\
& 11 a 15 años & 29 & 14 \\
& 16 a 20 años & 17 & 9.9 \\
Clase de vinculación & 21 a 25 años & 12 & 9.9 \\
& Más de 25 años & 12 & 9.1 \\
\hline & Empleado & 11 & 37.2 \\
& Independiente & 45 & 16.5 \\
& Ambas & 20 & 46.3 \\
\hline
\end{tabular}

Fuente: elaboración propia.

bles en cada institución de velar por la salud mental de sus empleados.

\section{Método}

\section{Muestra}

La población estuvo constituida por médicos psiquiatras egresados de programas nacionales y extranjeros, que ejercen la profesión en Colombia y que se encuentran registrados en la Asociación Colombiana de Psiquiatría (ACP), aunque no fueran miembros activos. El marco muestral estuvo constituido inicialmente por el listado de psiquiatras con sus correspondientes correos electrónicos, cuyos datos estuvieran en la ACP.

En total se pudo construir una base de datos de 631 psiquiatras, de la que debieron excluirse 217 personas, ya que al enviar los respectivos mensajes sus correos aparecían como inactivos, no existentes, o se informaba que el mensaje no pudo ser enviado. De las 414 personas restantes se obtuvo respuesta de 131, y de éstas 7 se encontraban fuera del país, 2 no deseaban participar en el estudio y 1 no era psiquiatra, de manea que la muestra final estuvo conformada por 121 personas.

Sus edades estaban en un rango entre 27 y 82 años, con un promedio de $40.94(D E=9.9)$. La Tabla 1 expone las características demográficas de la muestra de participantes, quienes pertenecían a 22 ciudades y municipios del país. La mayoría trabajaban en Bogotá (54\%).

\section{Instrumentos}

\section{Cuestionario para la Evaluación} del Síndrome de Quemarse por el Trabajo (CESQT) (Gil-Monte Ė Moreno-Jiménez, 2007)

Este instrumento está formado por 20 ítems que se distribuyen en 4 dimensiones denominadas: Ilusión 
por el trabajo (5 items) $(a l f a=0.9)$, Desgaste psíquico (4 items) $(\mathrm{alfa}=0.84)$, Indolencia (6 items) $($ alfa $=0.66)$ y Culpa (5 items) (alfa $=0.79)$ (GilMonteet al., 2005). La consistencia interna para la escala de 15 items (excluida la subescala de Culpa) ha reportado un valor alfa $=0.87$. (Gil-Monte et al., 2005). Los ítems se evalúan mediante una escala Likert de 5 grados que van de 0 (Nunca) a 4 (Muy frecuentemente: todos los días).

Según los autores, "este instrumento y sus dimensiones ha alcanzado valores de consistencia interna Alfa de Cronbach superiores a 0.70 en todos los estudios" (Gil-Monte \& Olivares, 2007). En la muestra de este estudio los datos de confiabilidad obtenidos fueron los siguientes: Ilusión por el trabajo alfa $=0.83$, Desgaste psíquico alfa $=0.88$, Indolencia alfa $=0.77$, y Culpa alfa $=0.86$.

Según el cuestionario se considera que la persona tiene burnout cuando presenta altas puntuaciones en el CESQT (puntuación mayor a 2), excluida la escala de Culpa. Se considera un caso grave si la persona presenta bajas puntuaciones en Ilusión por el trabajo, junto con altas puntuaciones en Desgaste psíquico e Indolencia. El caso será muy grave cuando altas puntuaciones en el CESQT se acompañan de altos sentimientos de Culpa.

Los resultados sobre validez factorial también han podido establecer un paralelo entre las escalas del CESQT y las de otros cuestionarios que evalúan el burnout. Considerando el MBI, la escala de Desgaste psíquico mide un constructo similar a Agotamiento; la escala de Indolencia mide un constructo similar a Despersonalización, y la escala de Ilusión por el trabajo mide un constructo parcialmente similar a Realización personal en el trabajo (Gil-Monte et al., 2006).

\section{Información demográfica y laboral}

Las variables a explorar fueron:

1. Sexo (hombre, mujer).

2. Edad (en años cumplidos).

3. Ciudad (de residencia).

4. Tiempo de ejercicio de la profesión (se mide en rangos por años, que permitirá evaluar el tiempo de experiencia).

5. Clase de vinculación (trabajo como empleado en una institución, independiente o ambas formas de vinculación).

6. Tipo de actividades que realiza (administrativas, docencia, investigación o clínica).

\section{Procedimiento}

Inicialmente se envió una solicitud escrita de la investigadora principal a la Asociación Colombiana de Psiquiatría. Después de la aprobación del proyecto la Asociación envió una base de datos con los correos electrónicos de los psiquiatras, y posteriormente a este listado se agregaron otros correos electrónicos encontrados en bases de datos proporcionadas por otras fuentes (laboratorios farmacéuticos, universidades, referidos por personas participantes), realizándose la ubicación de los psiquiatras a partir del listado de correos electrónicos de dichas bases de datos. Aquellas personas que contaban con más de un correo electrónico recibieron la información en todos ellos.

La elección de la recolección de los datos por correo electrónico se escogió por tres motivos: a) poder acceder a la mayor cantidad de personas en diferentes ciudades del país; b) garantizar la confiabilidad, y c) facilitar el acceso directo de las respuestas a una base de datos evitando errores de digitación. Los correos fueron enviados a cada uno de los participantes y en ellos se explicaba el objetivo del estudio, y se invitaba a ingresar a un link que contenía un código para cada uno, el cual enviaba a una página donde aparecía el consentimiento informado. Sólo en caso que el consentimiento fuera aceptado aparecía una nueva página con el formulario. Una vez los participantes llenaban el formulario y oprimían aceptar, sus respuestas automáticamente quedaban digitadas en una base de datos construida para tal fin, consignadas con el código que correspondía a cada uno de los participantes, de forma tal que los datos no pudieran ser identificados con nombre propio, lo cual garantizaba la confidencialidad. Los psiquiatras que no contestaron con el primer 
correo electrónico enviado recibieron un segundo correo invitándolos nuevamente a participar, y con quienes aceptaron se realizó inicialmente una primera fase de aplicación del Cuestionario para la Evaluación del Síndrome de Quemarse por el Trabajo (SQT) para establecer la prevalencia de burnout.

A partir de estos resultados se construyó una base de datos y se identificaron dos grupos: psiquiatras que presentaban burnout y psiquiatras que no lo presentaban, según el instrumento de medición del síndrome. Se obtuvieron resultados descriptivos de las características demográficas de cada grupo, se realizaron análisis de correlación para evaluar la relación entre la edad y el burnout, y también se hicieron pruebas de regresión logística y lineal para evaluar la capacidad de algunas variables de discriminar entre los grupos con o sin burnout y para evaluar el porcentaje de varianza explicado por un grupo de variables que mostraron estar relacionadas con el síndrome. Los anteriores procedimientos se realizaron usando el programa estadístico SPSS versión 13.

\section{Resultados}

\section{Prevalencia de burnout}

Para contestar la pregunta del estudio respecto a la prevalencia del burnout en los psiquiatras de la muestra se siguió el procedimiento de los puntos de referencia de la escala Likert con la que los sujetos valoran los ítems del cuestionario (Gil-Monte et al., 2005). La corrección de esta escala se realizó promediando el puntaje obtenido en cada una de las sub-escalas y de las 3 escalas en total (Ilusión, Desgaste, Indolencia). Los promedios mayores o iguales a 2 se consideran altos, y los menores a 2, bajos. La ilusión por el trabajo funciona como una variable inversa, es decir que niveles altos de ilusión se relacionan con menor burnout (Tabla 2).

Siguiendo ese procedimiento, se estableció que 7 personas (5.8\%) percibían "Ilusión por el trabajo" con una puntuación inferior a 2 (niveles bajos), 30 (24.8\%) percibían "Desgaste psíquico" con una puntuación igual o superior a 2 (niveles altos), y $10(8.3 \%)$ percibía niveles altos de "Indolencia". Respecto a la variable "Culpa", 8 personas (6.6\%) experimentaban sentimientos de culpa con niveles altos (mayor o igual a 2).

Cuando se consideró la puntuación total promediada en los 15 ítems, el número de personas que percibió niveles altos de burnout, con un puntaje mayor o igual a 2, fue 12 (9.9\%), pero de estas personas sólo 6 (4.96\%) presentaron, además, altas puntuaciones (mayores a 2) en las tres dimensiones del CESQT y 1 presentó altas puntuaciones en las 3 dimensiones y en la dimensión Culpa. Según estos resultados, $9.9 \%$ de los participantes percibieron niveles suficientemente altos de burnout para determinar que están afectados por este problema. Según las recomendaciones originales de los autores de la escala, dichas personas pueden ser categorizadas como de alto riesgo, y es recomendable la intervención para prevenir un mayor deterioro. Sólo 4.96\% de ellas se consideran "casos graves", pues presentan bajas puntuaciones en Ilusión por el trabajo, junto con altas puntuaciones en Desgas-

TABLA 2

Número y porcentaje de personas con altos y bajos niveles de burnout

\begin{tabular}{lcc}
\hline & Niveles altos $\geq 2$ & Niveles bajos $<2$ \\
\hline Ilusión por el trabajo & $114(94.2 \%)$ & $7(5.8 \%)$ \\
Desgaste psíquico & $30(24.8 \%)$ & $91(75.2 \%)$ \\
Indolencia & $10(8.3 \%)$ & $111(91.7 \%)$ \\
Culpa & $8(6.6 \%)$ & $113(93.4 \%)$ \\
CESQT-PD (15 ítems) & $12(9.9 \%)$ & $109(90.1 \%)$ \\
3 dimensiones CESQT-PD & $6(4.96 \%)$ & $115(95.04 \%)$ \\
CESQT-PD y culpa & $1(0.8 \%)$ & $120(99.17 \%)$ \\
\hline
\end{tabular}

Fuente: elaboración propia. 
te psíquico e Indolencia. Sólo 1 persona se ajusta al diagnóstico de "caso muy grave", pues presenta altas puntuaciones en el CESQT junto con altos sentimientos de Culpa.

Como se mencionó en el apartado sobre medición en burnout, es importante contar con puntos de corte para cada población, que permitan utilizar el instrumento para realizar un diagnóstico. Siguiendo el criterio sugerido por el autor de la escala a fin de dividir las puntuaciones de la muestra en tres grupos (bajo, medio y alto), para refinar el diagnóstico se calcularon los puntos de corte para los percentiles 33 y 66, pero también para los percentiles 10, 25, 75, y 90 (ver Tablas 3 y 4, en las cuales se especifican las puntuaciones que pueden utilizarse para efectos diagnósticos).

\section{TABLA 3}

Valores de los puntos de corte para los percentiles 33 y 66 obtenidos para las escalas del CESQT con la muestra del estudio

\begin{tabular}{lcc}
\hline & P33 & P66 \\
\hline Ilusión & 3.05 & 3.80 \\
Desgaste & 1 & 1.75 \\
Indolencia & 0.66 & 1.08 \\
Culpa & 0.40 & 1 \\
CESQT & 0.66 & 1.06 \\
\hline
\end{tabular}

Fuente: elaboración propia.

TABLA 4

Valores de los percentiles 10, 25, 75 y 90 obtenidos para las escalas del CESQT con la muestra del estudio

\begin{tabular}{lcccc}
\hline & P10 & P25 & P75 & P90 \\
\hline Ilusión & 2.04 & 2.80 & 4 & 4 \\
Desgaste & 0.25 & 0.75 & 1.87 & 2.75 \\
Indolencia & 0.33 & 0.50 & 1.16 & 1.80 \\
Culpa & 0 & 0.20 & 1 & 1.60 \\
CESQT & 0.34 & 0.53 & 1.30 & 1.97 \\
\hline
\end{tabular}

Fuente: elaboración propia.

Analizando los casos mediante el procedimiento de percentiles, se obtuvo que los porcentajes más similares a los obtenidos con el procedimiento de la escala Likert correspondían a los casos que se situaban por encima del percentil 90, excepto para la escala
Desgaste psíquico que resultó ser el percentil 75. Según esto, 109 personas (90.08\%) tenían altos niveles de Ilusión, 30 (24.8\%) altos niveles de Desgaste, 12 (9.9\%) altos niveles de Indolencia y 13 (10.74\%) altos niveles de Culpa. Al mirar la puntuación total del CESQT, 12 de las personas (9.9\%) percibieron niveles suficientemente altos de burnout, determinando que están afectadas por este problema.

\section{Factores de riesgo demográficos y laborales asociados a burnout}

Con el total de la muestra (121 personas) se realizó un análisis de los datos con el método de regresión logística para evaluar si las variables estudiadas permitían predecir la presencia o no de burnout. Las variables independientes incluidas fueron edad, sexo, estado civil, tiempo de ejercicio de la profesión, clase de vinculación y tipo de actividades que realiza (clínica, docencia, investigación, administrativa), pero no fue posible discriminar, a partir de las variables mencionadas, la presencia o no del burnout. El modelo de regresión logística planteado indicó que ninguna de las variables predijo de forma significativa la presencia del síndrome.

La variable burnout también puede analizarse como una variable continua, teniendo en cuenta el puntaje total obtenido por cada uno de los participantes en la escala. Para examinar la asociación entre la edad (variable continua) con el nivel de burnout se calculó una correlación de Pearson, la cual fue significativa y negativa $(r=-0.26 ; p<0.05)$, e indicó que a mayor edad los psiquiatras estudiados presentaron menos burnout.

El análisis de regresión lineal permitió examinar la explicación de la variabilidad del burnout teniendo como variables independientes sexo, estado civil, tiempo de ejercicio, tipo de vinculación y tipo de actividad. El modelo resultó significativo y explica el $17 \%$ de la varianza del burnout $\left(R^{2}=0.17\right.$; $\left.F_{(6)}=3.89 ; p<0.01\right)$. Las variables comparativamente más importantes fueron el trabajo exclusivo en el área clínica $(\beta=0.19 ; p<0.05)$ y la vinculación como empleado $(\beta=0.19 ; p<0.05)$. Las otras variables no mostraron suficiente capacidad explicativa. 


\section{Discusión}

Según los resultados de este trabajo un 9.9\% de los psiquiatras presentaron altos niveles de burnout, y de ellos 4.96\% eran casos graves. Si bien en la literatura se reportan prevalencias que oscilan entre un $30 \%$ y un $50 \%$ en médicos (Illera, 2006), es importante aclarar que dichos estudios han sido realizados con el Maslach Burnout Inventory (MBI). Como se mencionó anteriormente, este instrumento presenta dificultades de medición cuando se utiliza para establecer prevalencias. Para algunos autores las cifras que concluyen que la prevalencia se sitúa en porcentajes del $30 \%$ son desmesuradas y debidas a una utilización incorrecta del instrumento, y consideran que los datos más fiables sitúan los porcentajes alrededor del 10\% (Marucco, Gil-Monte \& Flamenco, 2008). Estudios de prevalencia realizados con el Cuestionario para la Evaluación del Síndrome de Quemarse por el Trabajo (CESQT) han mostrado prevalencias que oscilan entre el $3.25 \%$ y el $11.7 \%$, rango dentro del cual se encontrarían los participantes de este estudio, lo que significa que de los 1.098 psiquiatras que había en Colombia en el 2003 (Gómez-Restrepo, Bohórquez, Felizzola, DíazGranados \& Heins, 2003), 109 estarían afectados con burnout, cifra bastante importante si pensamos que el impacto del síndrome no lo padecen sólo los directamente afectados, sino los pacientes y familiares de cada una de estas personas.

Más allá del dato sobre la prevalencia, el análisis de los componentes del síndrome brinda también aportes interesantes. Si bien hay un porcentaje significativo de personas que auto reportan sentir Desgaste (24.8\%), es muy alto el porcentaje de aquellos que presentan altos niveles de Ilusión por su trabajo (94.2\%). Quienes han definido y estudiado el burnout afirman que éste es un fenómeno de naturaleza dinámica (va cambiando con el tiempo) y progresiva (avanza en fases), donde la pérdida por la Ilusión por el trabajo y el aumento en el Desgaste psíquico aparecen en un primer momento como respuesta al estrés laboral crónico, y donde posteriormente se desarrollan actitudes negativas hacia las personas que atienden en su trabajo. Podría pensarse entonces que los altos niveles de ilusión y satisfacción actúan como contrapeso para evitar la progresión hacia el síndrome a pesar del desgaste.

En este estudio se encontró que había una mayor asociación entre ser joven y presentar burnout. La edad como un factor de riesgo es un hallazgo que ha sido descrito en otras publicaciones (Atance, 1997), incluso en estudios con psiquiatras (Benbow $\&$ Jolley, 2002). Al respecto se han planteado varias explicaciones. Para Atance (1997):

“(...) puede existir un periodo de sensibilización debido a que habría unos años en los que el profesional sería especialmente vulnerable a éste, siendo éstos los primeros años de carrera profesional, dado que sería el período en el que se produce la transición de las expectativas idealistas hacia la práctica cotidiana, aprendiéndose en este tiempo que tanto las recompensas personales, profesionales y económicas, no son ni las prometidas ni las esperadas" (p. 294).

Cuando los psiquiatras inician su vida laboral están más expuestos a aceptar trabajos por razones estratégicas que por las recompensas que ofrecen. Es posible que en la medida que se adquiere más experiencia se pueda optar por trabajos con mayor posición, mayor control y mejores salarios. Otro fenómeno que puede explicar los hallazgos en relación con la edad es el de "supervivencia": aquellos que desarrollan altos niveles de burnout es más probable que hayan abandonado la profesión (Benbow \& Jolley, 2002). En un estudio realizado en infectólogos (citado por Deckard, Meterko \& Field, 1994) se encontró que entre los médicos que expresaron su intención de dejar el trabajo, el 58.9\% tenían altos niveles de agotamiento emocional. Otras hipótesis en cuanto a la edad se refieren a los métodos de afrontamiento. En el estudio realizado por Deckard, Meterko y Field (1994) se encontró que los médicos más jóvenes presentaban niveles más altos de agotamiento emocional que los de mayor edad, aduciendo que esto se debe a que los mecanismos de afrontamiento en el trabajo tienen un periodo crítico de ajuste a la situación laboral que puede durar los primeros años de vida profesional.

Otro hallazgo relevante del presente estudio es la mayor presencia de burnout en aquellos psi- 
quiatras que trabajan exclusivamente en el área clínica, en comparación con aquellos que poseen además actividades docentes, administrativas o de investigación, lo que también coincide con hallazgos de otras investigaciones donde los psiquiatras que tenían más tiempo para investigar o estudiar tenían niveles más bajos de burnout (Benbow \& Jolley, 2002). La psiquiatría se considera una especialidad altamente estresante en comparación a otras especialidades médicas por su naturaleza emocionalmente demandante (Fischer, Kumar \& Hatcher, 2007). Si bien la aparición del burnout va ligada directamente a la práctica clínica por implicar cuidado de otros y una alta carga emocional (factores de riesgo), podría pensarse a manera de prevención que los lugares de trabajo deben facilitar a las personas la posibilidad de cubrir otros intereses de su trabajo, que además garanticen tiempo para la investigación, la docencia y la formación, lo que se apoya también en los resultados de un trabajo realizado con psiquiatras en el que el trabajo académico y de investigación mostraron ser factores protectores para la aparición de burnout (Fischer, Kumar \& Hatcher, 2007).

Además de las implicaciones que pueda tener este trabajo a nivel del mejoramiento de la profesión, también debe destacarse el uso por primera vez en Colombia del instrumento del CESQT, demostrando adecuadas propiedades psicométricas que permitirían que fuera utilizado en otros estudios en el país.

Dentro de las limitaciones de este estudio se encuentra que la tasa de respuesta fue relativamente baja, aunque se ubica dentro del rango de lo hallado en otros trabajos similares (Mears et al., 2004). Los datos cubren un amplio rango de aquellos que trabajan como psiquiatras, y en cuanto a la distribución por ciudades de los participantes corresponde con la distribución porcentual por regiones de psiquiatras de todo el país registrados en la Asociación Colombiana de Psiquiatría (GómezRestrepo, Bohórquez, Felizzola, Díaz-Granados $\&$ Heins, 2003). Aún así estos resultados tienen limitaciones en su generalización, dado que no es posible establecer diferencias con aquellos que no respondieron. Otra de las limitaciones se encuentra en la metodología utilizada para la recolección de la información, pues al no realizarse un muestreo probabilístico es posible que personas que podrían tener burnout, por las mismas características de su cuadro, no encontraran motivante la participación en el estudio. Para futuras investigaciones se sugiere además la inclusión de otras variables que al parecer tienen una participación importante como la satisfacción laboral. De igual forma se sugiere el diseño y evaluación de programas de prevención y manejo.

\section{Referencias}

Atance, J. C. (1997). Aspectos epidemiológicos del síndrome de burnout en personal sanitario. Revista Española de Salud Pública, 71, 293-303.

Benbow, S. M. \& Jolley, D. J. (2002). Burnout and stress amongst old age psychiatrists. International Journal of Geriatric Psychiatry, 17, 710-714.

Cebrià-Andreu, J. (2005). Comentario: el síndrome de desgaste profesional como problema de salud pública. Gaceta Sanitaria, 19 (6), 463-70.

Deckard, G., Meterko, M. \& Field, D. (1994). Physician Burnout: An Examination of Personal, Professional, and Organizational Relationships. Medical Care, 32(7), 745-754.

Fischer, J., Kumar, S. \& Hatcher, S. (2007). What makes psychiatry such a stressful profession? A qualitative study. Australasian Psychiatry, 15(5), 417-421.

Gil-Monte, P. R., Carretero, N., Roldán, M. D. \& NúñezRomán, E. M. (2005). Prevalencia del síndrome de quemarse por el trabajo (burnout) en monitores de taller para personas con discapacidad. Revista de Psicología del Trabajo y de las Organizaciones, 21(1-2), 107-123.

Gil-Monte, P. R., García-Juesas, J. A., Núñez, E., Carretero, N., Roldán, M. D. \& Caro, M. (2006). Validez factorial del "Cuestionario para la Evaluación del Síndrome de Quemarse por el Trabajo" (CESQT). Recuperado el 25 de octubre de 2007, del sitio web de INTERPSIQUIS: http://www.psiquiatria.com/ articulos/estres/24872/

Gil-Monte,P.R. \&Moreno-Jiménez,B. (2007).Elsíndromede quemarse por el trabajo (burnout): grupos profesionales de riesgo. Madrid: Pirámide. 
Gil-Monte, P. R. \& Olivares, V. (2007). Prevalencia del Síndrome de Quemarse por el Trabajo (SQT) (burnout) en profesionales de atención a clientes de Chile. CD de comunicaciones del "5oㅡ Congreso Internacional de Prevención de Riesgos Laborales". Santiago de Chile (Chile).

Gómez-Restrepo, C., Bohórquez, A. P., Felizzola, C., Gil, F., Díaz-Granados, N. \& Heins, E. (2003). El perfil del médico psiquiatra colombiano. Revista Colombiana de Psiquiatría, 32(4), 325-340.

Guevara, C. A., Henao, D. P. \& Herrera, J. A. (2004). Síndrome de desgaste profesional en médicos internos y residentes. Hospital Universitario del Valle, Cali, 2002. Colombia Médica, 35(4), 173-178.

Halbesleben, J. R. \& Demerouti, E. (2005). The construct validity of an alternative measure of burnout: Investigating the English translation of the Oldenburg Burnout Inventory. Work $\mathcal{B}$ Stress, 19(3), 208-22.

Illera, D. (2006). Síndrome de burnout, aproximaciones teóricas. Resultado de algunos estudios en Popayán. Recuperado el 21 de marzo de 2007 del sitio web de la Facultad de Salud de la Universidad del Cauca: http://www.facultadsalud.unicauca.edu.co/ fcs/2006/septiembre/BOURNOT.pdf

Korkeila, J. A., Töyry, S., Kumpulainen, K., Toivola, J. \& Räsänen, K. (2003). Burnout and self-perceived health among Finnish psychiatrists and child psychiatrists: a national survey. Scandinavian Journal of Public Health, 31, 85-91.

Kristensen, T. S., Borritz, M., Villadsen, E. \& Christensen, K. B. (2005). The Copenhagen Burnout Inventory: A new tool for the assessment of burnout. Work EZ Stress, 19(3), 192-207.

Maslach, C. (2003). Job Burnout: New Directions in Research and Intervention. Current Directions in Psychological Science, 12(5), 189-192.

Maslach, C. \& Leiter, M. P. (2003). Areas of worklife: a structured approach to organizational predictors of job burnout. En P. L. Perrewe \& D. C. Ganster (Eds.), Emotional and Physiological Processes and Positive Intervention Strategies. Vol. 1. Emerald Group Publishing.

Marucco, M. A., Gil-Monte, P. R. \& Flamenco, E. (2008). Síndrome de quemarse por el trabajo (burnout) en pediatras de hospitales generales, estu- dio comparativo de la prevalencia medida con el MBI-HSS y el CESQT. Informació psicològica, 91-92, 32-42.

Mears, A., Pajak, S., Kendall, T., Katona, C., Medina, J., Huxley, P., et al., (2004). Consultant psychiatrists' working patterns: is a progressive approach the key to staff retention? Psychiatric Bulletin, 28, 251-253.

Noblet, A. (2003). Building health promoting work settings: identifying the relationship between work characteristics and occupational stress in Australia. Health Promotion International, 18(4), 351-359.

Olivares, V. E. \& Gil-Monte, P. R. (2007). Prevalencia del síndrome de quemarse por el trabajo (burnout) en trabajadores de servicios en Chile. Informació psicològica, 91, 43-52.

Restrepo, N. C., Colorado, G. O. \& Cabrera, G. A. (2006). Desgaste Emocional en Docentes Oficiales de Medellín, Colombia, 2005. Revista de Salud Pública, 8(1), 63-73.

Salston, M. D. \& Figley, C. R. (2003). Secondary Traumatic Stress Effects of Working with Survivors of Criminal Victimization. Journal of Traumatic Stress, 16(2), 167-174.

Spear, J., Wood, L., Chawla, S., Devis, A. \& Nelson, J. (2004). Job satisfaction and burnout in mental health services for older people. Australasian Psychiatry, 12(1), 58-61.

Trippany, R. L., White, V. E. \& Wilcoxon, S. A. (2004). Preventing Vicarious trauma: what counselors should know when working with trauma survivors. Journal of Counseling $\mathcal{E}$ development, 82, 31-37.

Unda, S., Sandoval, J. I. \& Gil-Monte, P. R. (2008). Prevalencia del Síndrome de Quemarse por el Trabajo (SQT) (burnout) en maestros mexicanos. Informació psicològica, 91-92, 53-63.

Visser, M. R., Smets, E. M., Oort, F. J. \& De Haes, H. C. (2003). Stress, satisfaction and burnout among Dutch medical specialists. Canadian Medical Association Journal, 68(3), 271-275.

Weber, A. \& Jaekel-Reinhard, A. (2000). Burnout syndrome: a disease of modern societies? Occupational Medicine, 50, 512-517.

White, P., Edwards, N. \& Townsend-White, C. (2006). Stress and burnout amongst professional carers of people with intellectual disability: another health inequity. Current Opinion in Psychiatry, 19, 502-507. 
